\title{
Modelisation Des Changements D'occupation Des Terres En Region Soudanienne Au Nord-Ouest Du Benin
}

\begin{abstract}
Agbanou Thierry,
Laboratoire de Biogéographie et Expertise Environnementale, Université d'Abomey-Calavi (LABEE/UAC/Bénin), Géographie de l'Environnement, Université Toulouse Jean Jaurès (GEODE/Toulouse 2 / France)
\end{abstract}

Paegelow Martin,

Géographie de l'Environnement,

Université Toulouse Jean Jaurès (GEODE/Toulouse 2 / France)

Toko Imorou Ismaïla,

Laboratoire de Cartographie, Université d'Abomey-Calavi (LaCarto/UAC/ Bénin)

\section{Tente Brice,}

Laboratoire de Biogéographie et Expertise Environnementale,

Université d'Abomey-Calavi (LABEE/UAC/Bénin)

\section{Doi: 10.19044/esj.2018.v14n12p248 URL:http://dx.doi.org/10.19044/esj.2018.v14n12p248}

\begin{abstract}
In north-west Benin, land occupation is undergoing a significant dynamic due to intense human activities putting the ecosystem in precarious balance. The objective of this study is to quantify landscape dynamics and explore possible futures of land use using remote sensing, GIS and spatiotemporal modelling tools. Landsat socio-economic data and images (1987, 2000 and 2016) were used. A multi-date classification was used to quantify changes in land use. The Land Change Modeler (LCM) of the Terrset software was used to simulate and project land use to 2031. The results show that between 1987 and 2016, the landscape initially dominated by open wooded forests and savannahs $(38,8 \%)$ and wooded and shrubby savannahs $(25,8 \%)$ in 1987 was replaced in 2016 by land use categories including wooded and shrubby savannahs (40,5\%) and crop and fallow mosaics (50,6\%). The three scenarios Business-As-Usual (BAU), Rapid Economic Growth (CER) and Coordinated Environmental Sustainability (CED), extrapolating the current trends, show that by 2031 this landscape will be dominated by crop and fallow mosaics $(+50 \%)$. Wood resources occupy $26 \%$ of the area of the sector in the CED scenario, $23 \%$ in the BAU scenario and 19\% in the CER scenario.
\end{abstract}


The CED scenario gives hope that the restoration and preservation of plant resources is still possible if decision-makers become aware of it.

Keywords: Cartography, Land-Use and Land-Cover Change, explanatory factors, modelling, Benin

\section{Résumé}

$\mathrm{Au}$ nord-ouest du Bénin, l'occupation des terres connaît une dynamique importante du fait des activités humaines intenses mettant l'écosystème en équilibre précaire. L'objectif de cette étude est de quantifier la dynamique paysagère et d'explorer les futurs possibles de l'occupation des terres à partir des outils de télédétection, de SIG et de la modélisation spatiotemporelle. Les données socio-économiques et images Landsat (1987, 2000 et 2016) ont été utilisées. Une classification multi-date a permis de quantifier les changements d'occupation des terres. Le modèle Land Change Modeler $(\mathrm{LCM}) \mathrm{du}$ logiciel Terrset a permis de simuler et de faire des projections à l'horizon 2031 de l'occupation des terres. Il ressort des résultats qu'entre 1987 et 2016, le paysage initialement dominé par les forêts claires et savanes boisées (38,8\%) et les savanes arborées et arbustives $(25,8 \%)$ en 1987 a été remplacé en 2016 par des catégories d'occupation des terres dont les savanes arborées et arbustives (40,5\%) et les mosaïques de cultures et jachères $(50,6 \%)$. Les trois scenarios Business-As-Usual (BAU), Croissance Economique Rapide (CER) et Durabilité Environnementale Coordonnée (DEC), extrapolant les tendances actuelles, montrent qu'à l'horizon 2031 ce paysage sera dominé par les mosaïques de cultures et jachères $(+50 \%)$. Les ressources ligneuses occupent $26 \%$ de la superficie du secteur dans le scénario DEC, $23 \%$ dans le scénario BAU et $19 \%$ dans le scénario CER. Le scénario DEC donne l'espoir que la restauration et la préservation des ressources végétales est encore possible si les décideurs en prennent conscience.

Mots clés : Cartographie, dynamique de l'occupation des terres, facteurs explicatifs, modélisation, Bénin

\section{Introduction}

Les terres dans le monde connaissent de profondes mutations spatiales. En Afrique de l'ouest et particulièrement au Bénin ces mutations spatiales qui se traduisent par la dégradation du couvert végétal s'accentuent de jour en jour. $\mathrm{Au}$ nord-ouest du Bénin, les savanes sont les plus importantes catégories d'occupation des terres. Elles sont des écosystèmes fragiles reposant sur la coexistence entre une strate herbacée et une strate ligneuse (Jacquin, 2010). Ces ressources subissent de fortes pressions anthropiques du fait de l'explosion démographique et d'un système cultural purement extensif. 
L'extension accrue des aires de cultures et des agglomérations engendre une réduction progressive de l'étendue des surfaces boisées (FAO, 2016) et une déstabilisation de la structure des sols (Akognongbé et al., 2014). La rotation des cultures et la jachère ont aussi des perturbations majeures sur ces systèmes écologiques (Faye et al., 2002) en équilibre précaire. Les changements d'usage des terres ont ainsi des répercussions directes sur l'occupation des terres et sur la configuration du paysage de ce milieu (Bamba et al., 2008). Ce changement de la configuration du paysage dépend de l'intensité et/ ou la durée des facteurs de pression.

Ainsi, la végétation est particulièrement sensible aux dynamiques de la composante anthropique, notamment l'agriculture itinérante sur brûlis, l'exploitation du bois et l'urbanisation croissante (Bigot et al., 2005) observées dans ce milieu. Face à cette dégradation continue du couvert végétal, on se demande quel est l'avenir du paysage de cette région?

Depuis quelques décennies, la modélisation et la projection de changement d'occupation des terres s'impose comme outil pertinent d'aide à la décision. Elle permet d'analyser les politiques de planification territoriale dans le but d'évaluer et d'anticiper leurs impacts environnementaux (Abdus et al., 2017). L'originalité de cette recherche réside dans le fait que la modélisation de la dynamique de l'occupation des terres permettra de suivre la tendance évolutive du paysage et de trouver des règles acceptables pour préserver les ressources naturelles, notamment les ressources ligneuses de ce milieu.

\section{Données et méthodes \\ Description de la zone d'étude}

Située dans le nord-ouest du Bénin, le secteur Natitingou-Boukombé est une partie de la chaîne de l'Atacora, le massif montagneux le plus important au Bénin. Il est situé entre les parallèles $10^{\circ} 00^{\prime}$ et $10^{\circ} 40^{\prime}$ de latitude nord et les méridiens $1^{\circ} 09^{\prime}$ et $1^{\circ} 46^{\prime}$ de longitude est (figure 1 ). Le climat est de type soudano-guinéen, qualifié d'atacorien (Houssou, 1998) caractérisé par une saison de pluie de 5 à 6 mois (mai à octobre) et une saison sèche de 6 à 7 mois (novembre à mai). Les moyennes pluviométriques sont de $1300 \mathrm{~mm}$ par an réparties sur 90 jours. La saison pluvieuse est la plus favorable au développement végétatif. L'agriculture itinérante sur brûlis est l'activité prépondérante pratiquée dans ce milieu. 


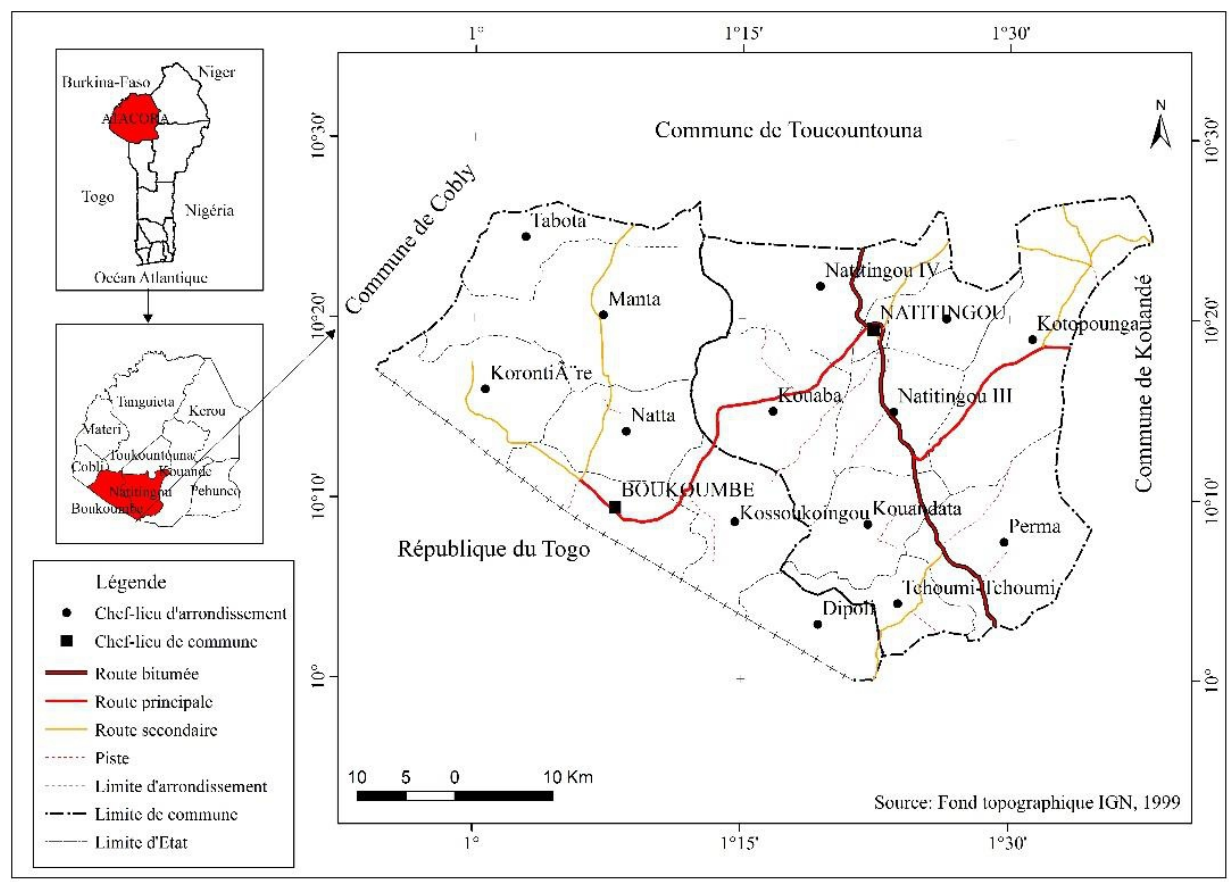

Figure 1 : Situation géographique et administrative du secteur Natitingou-Boukombé

\section{Données planimétriques}

Les données utilisées pour l'étude de la dynamique de l'occupation des terres ici sont essentiellement des images satellitaires Landsat TM du 23 janvier 1987, ETM + (Thematic Mapper) du 04 février 2000 et OLI- TIRS du 07 janvier 2016 de $30 \mathrm{~m}$ de résolution. Ces images ont été téléchargées gratuitement sur le site earthexplorer.usgs.gov au format GEOTIFF (Path 193 et Row 53). L'image SRTM de 2000 (résolution $90 \mathrm{~m}$ ) a été utilisée pour la réalisation des Modèles Numériques de Terrain (carte d'altitude et pente). Les cartes topographiques et fond de carte de l'Institut Géographique National (IGN) ont été également utilisés dans cette étude. Un GPS (Global Positioning System) de marque GARMIN a été utilisé pour localiser la position des différents points de contrôle terrain. A ces données planimétriques s'ajoutent quelques données socio-économiques (densité, superficies emblavées, pédologie, etc.) collectées sur le terrain et indispensables pour la simulation de l'occupation des terres. Le logiciel Terrset à travers son outil Land Change Modeler (LCM) a été utilisé pour la simulation de l'occupation des terres à l'horizon 2031.

\section{Méthodes}

\section{- Dynamique de l'occupation des terres}

Le logiciel Terrset a permis de réaliser trois cartes d'occupation des terres à partir des images satellitaires de 1987, 2000 et 2016. Ici les bandes 
radiométriques à même de nous fournir des informations sur le couvert végétal ont été superposées par synthèse additive des couleurs primaires. Ainsi la composition colorée en fausse couleurs avec la combinaison des bandes 234 pour les images TM/ETM+ et 345 pour l'images OLI TIR ont été utilisées. La méthode de classification supervisée selon l'algorithme du maximum de vraisemblance a été utilisée. La classification établie ainsi que les couleurs de représentation de chaque catégorie d'occupation des terres ont été adaptées de la nomenclature Corine Land Cover (CLC). En tout 150 points GPS ont été collecté le long de trois (03) transect, soit de 50 points par transect. Il s'agit d'un transect dans la zone à végétation développée, un autre dans la zone à végétation moyennement dégradée et un dernier dans la zone à végétation très dégradée. L'indice de Kappa a également permis de valider les classifications. Les cartes d'occupation des terres réalisées ont fourni les données de base pour l'analyse et la quantification du paysage. Les différents indices suivants ont été calculé pour mieux apprécier la dynamique observée :

\section{$\checkmark \quad$ Calcul du LUCC budget}

La matrice de transition des trois cartes d'occupation des terres (1987, 2000 et 2016) permet de faire le bilan des gains, des pertes et des persistances en termes de superficie des catégories d'occupation des terres entre deux dates différentes. Le gain brut pour chaque catégorie est calculé en soustrayant la persistance du total de la ligne, tandis que la perte brute est calculée en soustrayant la persistance du total de la colonne.

L'équation (1) et (2) donne la méthode de calcul du gain (G) et de la perte (L) observé au niveau de chaque catégorie d'occupation des terres entre deux dates.

$$
\begin{aligned}
G_{j} & =\frac{\left\{\left(\sum_{i=1}^{j} S j j\right)-S j j\right\} X 100}{\sum_{i=1}^{j} S i j} & \text { OU } & G_{j}=\left(\sum_{i=1}^{j} S_{j j}\right)-S_{j j} \\
L_{i} & =\frac{\left\{\left(\sum_{j=1}^{j} S i j\right)-S i i\right\} X 100}{\sum_{j=1}^{j} S i j} & \text { ou } & L_{i}=\left(\sum_{j=1}^{j} S_{i i}\right)-S_{i i}
\end{aligned}
$$

$\mathrm{G}_{\mathrm{j}}=$ Gain de superficie (ou en proportion) d'une catégorie d'occupation des terres $\mathrm{j}$

$\mathrm{L}_{\mathrm{i}}=$ Perte de superficie (ou en proportion) d'une catégorie d'occupation des terres $\mathrm{i}$

$\mathrm{S}_{\mathrm{ii}}=$ Superficie stable d'une catégorie d'occupation i entre deux dates

$\mathrm{S}_{\mathrm{jj}}=$ Superficie stable d'une catégorie d'occupation i entre deux dates

$\mathrm{S}_{\mathrm{ij}}=$ Superficie d'une catégorie d'occupation $\mathrm{i}$ transformée en $\mathrm{j}$ entre deux dates.

$\mathrm{S}_{\mathrm{ji}}=$ Superficie d'une catégorie d'occupation $\mathrm{j}$ transformée en $\mathrm{i}$ entre deux dates

L'équation (3) exprime le changement total. La superficie totale changée est calculée en additionnant toutes les entrées de la matrice puis en 
soustrayant les persistances.

$$
\text { Changement Total }=\frac{\sum_{j=1}^{j}\left\{\left(\sum_{i=1}^{j} S_{i j}\right)-S_{j j}\right\}}{\sum_{j=1}^{j} \sum_{i=1}^{j} S_{i j}}
$$

L’équation (4) indique comment calculer le changement net pour une catégorie $\mathrm{j}$ en ajoutant les transitions dans lesquelles la catégorie j gagne, c'està-dire $\mathrm{C}_{\mathrm{ij}}$, puis en soustrayant la transitions dans lesquelles la catégorie $\mathrm{j}$ perd, c'est-à-dire $C_{\mathrm{ji}}$. Autrement dit, pour une catégorie donnée, dans la matrice elle correspond à la différence entre le gain brut et la perte brute.

Changement Net $=\frac{\sum_{j=1}^{j}\left\{\operatorname{MAXIMUM}\left[0, \sum_{j=1}^{j}\left(S_{i j}-S_{j i}\right)\right]\right\}}{\sum_{j=1}^{j} \sum_{i=1}^{j} S_{i j}}$

L'équation (5) permet de calculer la balance qui est la différence entre le changement total et le changement net. Cette variable exprime un changement de lieu sans changement de quantité. Pontius et al. (2008) l'expliquent comme un désajustement qui pourrait être résolu en réorganisant les pixels d'une carte pour s'adapter autant que possible avec l'autre carte.

Swap $=$ Change Total - Changement Net

\section{$\checkmark \quad$ Calcul du taux d'évolution}

La dynamique de chaque catégorie d'occupation sera appréciée en calculant le taux d'évolution $\mathrm{E}_{(\mathrm{i}, \mathrm{k})}$ des superficies d'occupation des terres de la façon suivante :

Soit $\mathrm{S}_{\mathrm{i}}$ la superficie d'une catégorie d'occupation des terres de l'année $\mathrm{i}$ et $\mathrm{S}_{\mathrm{k}}$, celle d'une catégorie d'occupation des terres de l'année $\mathrm{k}$, avec $\mathrm{k}>\mathrm{i}$. $\mathrm{E}_{(\mathrm{i}, \mathrm{k})}$ sera égale à :

$$
E_{(i, k)}=\frac{S_{k}-S_{i}}{S_{i}} \times 100
$$

Si $E_{(i, k)}=0$, on conclut qu'il y a stabilité de cette catégorie d'occupation des terres ;

Si $E_{(i, k)}<0$, on conclut qu'il y a régression de cette catégorie ;

Si $E_{(i, k)}>0$, il y a extension ou évolution de cette catégorie.

\section{- Simulation de l'occupation des terres}

Le modèle de prédiction utilisé ici est Land Change Modeler (LCM) implémenté dans le logiciel Terrset (ex Idrisi). Ce modèle s'appuie sur les connaissances passées et actuelles de l'occupation des terres pour prédire le futur. Plusieurs facteurs influençant les changements d'occupation des terres ont été intégrés dans ce modèle. Il s'agit de la pente, l'altitude, la pédologie, la distance aux agglomérations, la distance aux champs, la distance aux plantations, la distance aux routes et la densité. L'indice V de Cramer a été calculé pour chaque facteur explicatif et a permis de retenir ceux qui contribuent le mieux à la mise en place de chaque catégorie d'occupation des terres. Les variables explicatives utilisées ici sont de deux types : dynamiques et statiques. Les variables statiques (altitude, pente, pédologie) traduisent 
l'aptitude pour chaque transition prise en considération et restent stables au cours du temps. Les variables dynamiques (proximité aux routes, proximité aux types d'occupation des terres déjà existants) sont recalculées à chaque pas de temps durant le processus de prédiction. La réalisation de la carte de changement de 2000-2016 a permis d'identifier les transitions majeures et de les regrouper en sous-modèles.

Les cartes de prédiction ont été élaborées par le réseau de neurones multicouche à partir des cartes de transitions observées et des variables explicatives. Ceux-ci assignent à chaque pixel une catégorie de détérioration à l'aide du processus de décision multi-objectif (MOLA). Pour attribuer la quantité de changement, l'algorithme s'appuie sur la matrice de probabilité de transition 2016-2031 générée.

Deux types de carte ont été générées à la sortie du modèle : une carte de simulation dite " hard », qui alloue à chaque pixel de la carte un type d'occupation des terres et un seul, et un résultat de simulation dit « soft», qui associe à chaque pixel une valeur d'intensité de changement au cours du temps, une sorte de propension générale au changement d'occupation des terres quelle qu'en soit la nature. Cette propension est calculée par cumul des probabilités de changement à chaque pas de la simulation.

\section{- Description des différents scénarios prospectifs}

Trois scénarios ont été élaborés pour prédire les tendances actuelles de changement et d'utilisation des sols afin de faciliter les prises de décisions. Il s'agit de :

\section{Scénario 1 : Business As Usual (BAU) extrapolant les tendances actuelles en 2031}

Le scénario BAU est un scénario tendanciel qui part de l'hypothèse de l'absence de nouvelles politiques économiques ou environnementales. C'est un scénario dans lequel les futures distributions d'utilisation des terres suivent les tendances observées dans le passé. Il se base sur les données socioéconomiques passées récentes (2000-2016), par exemple, la croissance économique et démographique. Le secteur n'attire pas l'arrivée de nouveaux habitants qui pourraient éventuellement perturber le système en place. Du coup, la demande en superficie à emblaver et la demande en bois de chauffage resteront dans une dynamique similaire à celle observée au cours de la période 2000-2016.

\section{Scénario 2 : Durabilité Environnementale Coordonnée (DEC) «Alafia 2031 "}

Le principal objectif du scénario Alafia est de protéger le reste des ressources végétales encore en place. Dans ce scénario, le gouvernement encourage la foresterie. C'est un scénario dans lequel la législation et les 
subventions gouvernementales encouragent l'émergence de la sylviculture (multiplication des plantations et de l'agroforesterie) et la protection des ressources ligneuses en subventionnant le prix du gaz pour qu'il soit accessible à tous les ménages ou en créant des plantations pour bois de feu à exploiter par les populations. Dans ce scénario les probabilités pour les savanes arborées et arbustives à se transformer en mosaïques de cultures en 2031 ont été modifiées à la hausse dans la matrice de transition. Tandis que celles des forêts denses, forêts claires et savanes boisées à se transformer en catégories plus dégradées (pauvres en ressources ligneuses) en 2031 ont été réduites de façon à protéger (favoriser la persistance) le couvert végétal arboré et arbustif encore présent. Aussi, les probabilités de transformation des savanes arborées et arbustives et des mosaïques de cultures et jachères à se transformer en plantations en 2031 ont été augmentées de façon à favoriser la sylviculture et le développement de l'agroforesterie

\section{Scénario 3 : Croissance Economique Rapide (CER) « Wahala 2031 »}

Le scénario Wahala est un scénario socio-économique, c'est-à-dire d'accélération de la destruction du couvert végétal arboré et arbustif et d'élargissement des terres agricoles (tendance à la catastrophe). Dans ce scénario, la croissance démographique et économique sera très élevée et se soldant par une dégradation accrue de l'environnement. C'est un scénario pessimiste, mais très probable dans ce milieu d'étude où il n'y a actuellement aucune politique de gestion durable des ressources naturelles. C'est un scénario dans lequel seules les superficies des mosaïques de cultures er jachères et les agglomérations ont été augmentées de façon considérable. Du coup, les probabilités de transformation des savanes arborées et arbustives en forêts denses et forêts claires et savanes boisées ont été baissées. Tandis que les probabilités des savanes arborées et arbustives, des forêts denses et forêts claires et savanes boisées à se transformer en mosaïques de cultures et jachères ont été augmentées.

\section{Résultats}

\section{Dynamique de l'occupation des terres en 1987-2000 et 2000-2016}

L'analyse des cartes d'occupations des terres de 1987, 2000 et 2016 ont permis de constater une différence dans la dynamique observée au cours des deux périodes 1987-2000 et 2000-2016. La figure 2 présente une synthèse des trois cartes d'occupation et des statistiques issues de l'analyse diachronique. 


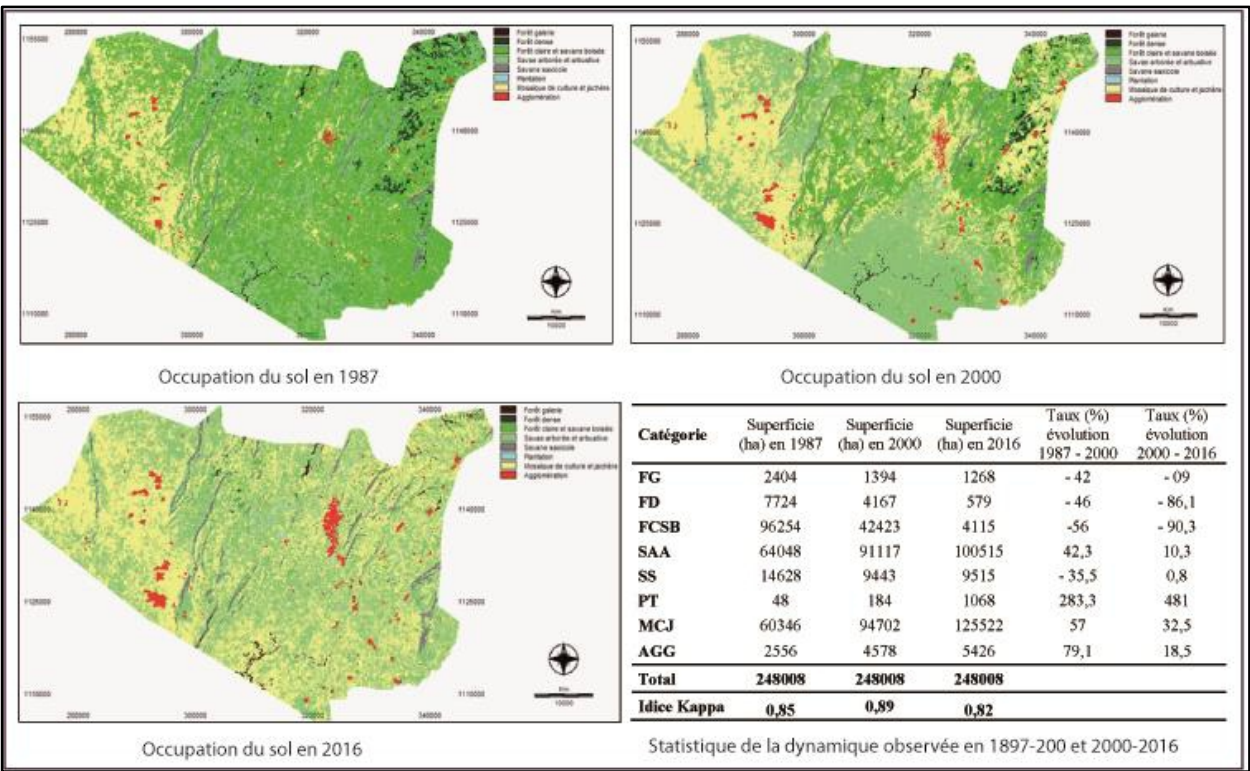

Légende : FG : Forêt galerie ; FD : Forêt dense ; FCSB : Forêt claire et savane boisée ;

SAA : Savanes arborée et arbustive ; SS : Savane saxicole ; PT : Plantation ; MCJ :

Mosaïque de Champs et jachères ; AGG : Agglomération

Figure 2 : Occupation des terres de 1987, 2000 et 2016

L'examen la figure 2 permet de constater que l'indice de Kappa pour chacune des trois classifications est supérieur à $75 \%$ (seuil recommandé). Ceci que ces cartes d'occupation des terres sont acceptables. En ce qui concerne la dynamique d'occupation des terres, les forêts denses ont connu une régression de $46 \%$ au cours de la période $1987-2000$ contre $86,1 \%$ pour la période 2000-2016. Les forêts claires et savanes boisées ont régressé de $56 \%$ au cours de la période 1987-2000 contre 90,3\% pour 2000-2016. Les savanes arborées et arbustives, les mosaïques de cultures et jachères, les agglomérations et les plantations ont connu respectivement une progression de $42,3 \% ; 57 \% ; 79,1 \%$ et $283,3 \%$ au cours de la période $1987-2000$ contre respectivement $10,3 \% ; 32,5 \% ; 18,5 \%$ et $481 \%$ au cours de la période 2000 2016. Les tableaux I et II présentent le LUCC budget (Land-Use and LandCover Change) calculé pour les deux périodes 1987-2000 et 2000-2016.

Tableau I : LUCC budget pour la période 1987-2000 (en ha)

\begin{tabular}{cccccc}
\hline Catégorie & Gain & Perte & $\begin{array}{c}\text { Changement } \\
\text { total }\end{array}$ & Balance & $\begin{array}{c}\text { Valeur absolue } \\
\text { changement net }\end{array}$ \\
\hline FG & 0 & 95,5 & 95,5 & 0 & 95,5 \\
\hline FD & 0 & 1,2 & 1,2 & 0 & 1,2 \\
\hline FCSB & 73564,4 & 20534 & 94098,3 & 41068 & 53030,4 \\
\hline SAA & 30081,6 & 57192,6 & 87274,2 & 60163,2 & 27111 \\
\hline SS & 1421,8 & 533,3 & 1955,1 & 1066,5 & 888,6 \\
\hline PT & 100 & 36,1 & 136,1 & 72,2 & 64 \\
\hline
\end{tabular}




\begin{tabular}{cccccc}
\hline MCJ & 23809,4 & 52830,5 & 76639,9 & 47618,8 & 29021,1 \\
\hline AGG & 2331 & 85,2 & 2416,2 & 170,5 & 2245,8 \\
\hline
\end{tabular}

Source : Interprétation images Landsat TM de 1987 et ETM+ de 2000

Légende : FG : Forêt galerie ; FD : Forêt dense ; FCSB : Forêt claire et savane boisée ; SAA : Savanes arborée et arbustive ; SS : Savane saxicole ; PT : Plantation ; MCJ : Mosaïque de Champs et jachères ; AGG : Agglomération

Tableau II : LUCC budget pour la période 2000-2016 (en ha)

\begin{tabular}{cccccc}
\hline Catégorie & Gain & Perte & $\begin{array}{c}\text { Changement } \\
\text { total }\end{array}$ & Balance & $\begin{array}{c}\text { Valeur absolue } \\
\text { changement net }\end{array}$ \\
\hline FG & 0 & 123,8 & 123,8 & 0 & 123,8 \\
\hline FD & 0 & 4154,9 & 4154,9 & 0 & 4154,9 \\
\hline FCSB & 1646,6 & 39430,2 & 41076,7 & 3293,1 & 37783,6 \\
\hline SAA & 52902 & 43235,7 & 96137,7 & 86471,5 & 9666,3 \\
\hline SS & 140,4 & 385,4 & 525,8 & 280,8 & 245 \\
\hline PT & 885,7 & 04,8 & 890,5 & 09,54 & 880,9 \\
\hline MCJ & 67518 & 36608,2 & 104126,2 & 73216,4 & 30909,8 \\
\hline AGG & 1069,6 & 219,3 & 1288,9 & 438,7 & 850,2 \\
\hline
\end{tabular}

Source : Interprétation images Landsat ETM+ de 2000 et OLI TIRS 2016

Légende : FG : Forêt galerie ; FD : Forêt dense ; FCSB : Forêt claire et savane boisée ; SAA : Savanes arborée et arbustive ; SS : Savane saxicole ; PT : Plantation ; MCJ : Mosaïque de Champs et jachères; AGG : Agglomération

Il ressort des LUCC budget (1987-2000 et 2000-2016) que les formations végétales naturelles comme les savanes arborées et arbustives, les forêts denses, forêts claires et savanes boisées ont perdu des superficies au profit des mosaïques de cultures et jachères, plantations et agglomérations. On observe, en outre, que pertes et gains des savanes arborées et arbustives tendent à s'équilibrer pour l'essentiel. Bien qu'elle a gagné 27111 ha (19872000 ) et 9666,3 ha (2000-2016), l'essentiel de la dynamique spatio-temporelle consiste en un changement de localisation des savanes arborées et arbustives : ce qu'elles ont perdu à un endroit, elles l'ont gagné ailleurs. Il en va de même pour les mosaïques de cultures et de jachères. Au-delà d'une extension de 29021,1 ha (1987-2000) et 30909,8 ha (2000-2016), on remarque aussi une relocalisation importante de 52830,5 ha, et 36608,2 ha pour respectivement les période 1987-2000 et 2000-2016. Quant aux forêts claires et savanes boisées, elles perdent 20534 ha et gagnent en retour 73564,4 ha, des changements de localisation estimées à 53030,4 ha pour la période 1987-2000. Alors qu'au cours de la période 2000-2016 les changements de localisation sont estimés à 37783,6 ha.

Globalement on peut retenir que de 1987 à 2016, les formations végétales naturelles (forêts denses, forêts claires et savanes boisées) ont régressé au profit des formations anthropiques (mosaïques de cultures et jachères, agglomérations, plantations). Ces mutations spatiales qui s'expliquent par plusieurs raisons dont l'évolution démographique ne sont pas 
de nature à faciliter les simulations. En effet, on observe une dynamique très différente entre les deux périodes. Du coup, le choix de la période 1987-2000 pour le calibrage du modèle parait irréaliste. Se basant sur la tendance évolutive à la hausse de la population de 1979 à nos jours avec son corolaire de forte demande de terres cultivables, la période la plus récente (2000-2016) sera retenue pour l'entraînement du modèle et la simulation.

\section{Mise en ouvre de la simulation prospective de l'occupation des terres}

La combinaison de la matrice de transition adaptée aux différents scénarii Business As Usual, Alafia et Wahala avec les cartes de potentiel de transition et les facteurs explicatifs a permis d'obtenir les cartes de prédiction 2031 (figure 3) et les statistiques relatives à la superficie des catégories d'occupation des terres (tableau III).

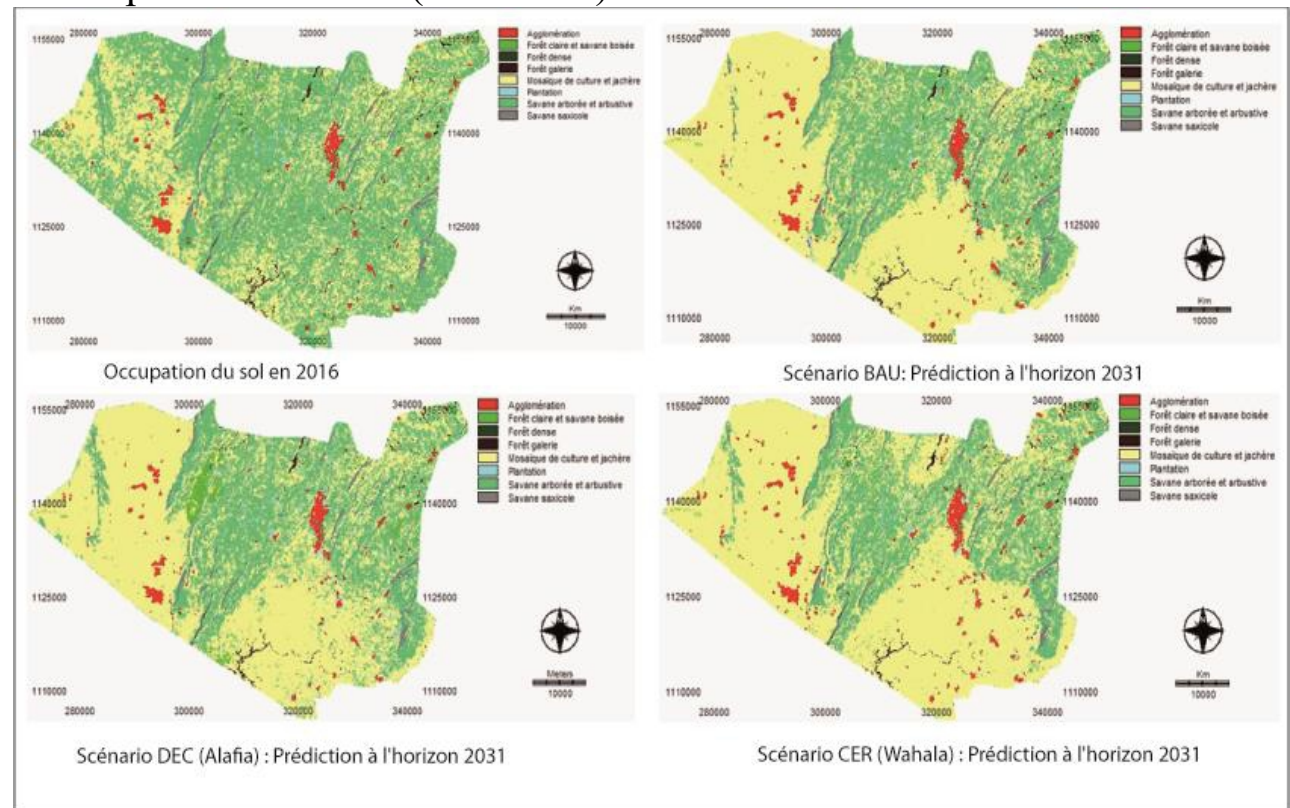

Figure 3 : Cartes d'occupation des terres de 2016 et celles simulées pour les trois scénarios BAU, DEC et CER

Tableau III : Statistiques des superficies de catégories d'occupation des terres en 2016 et 2031 avec les taux d'expansion

\begin{tabular}{ccccccccc}
\hline & $2016(\mathrm{ha})$ & $\%$ & BAU (ha) & Tx & DEC (ha) & Tx & CER (ha) & Tx \\
\hline FG & 1268 & 0,5 & 1268 & 0 & 1268 & 0 & 1268 & 0 \\
\hline FD & 579 & 0,2 & 187 & $-67,7$ & 387 & $-33,2$ & 187 & $-67,7$ \\
\hline FCSB & 4115 & 01,7 & 1989 & $-51,6$ & 4224 & 02,6 & 2220 & -46 \\
\hline SAA & 100515 & 40,5 & 54389 & $-45,9$ & 56062 & $-44,2$ & 43020 & $-57,2$ \\
\hline SS & 9515 & 03,9 & 9149 & 03,8 & 9186 & $-03,5$ & 9167 & $-03,7$ \\
\hline PT & 1068 & 0,4 & 1712 & 60,4 & 2616 & 145 & 1709 & 60,1 \\
\hline MCJ & 125522 & 50,6 & 172720 & 37,6 & 168575 & 34,3 & 182839 & 45,7 \\
\hline AGG & 5426 & 02,2 & 6594 & 21,5 & 5690 & 04,9 & 7598 & 40 \\
\hline
\end{tabular}




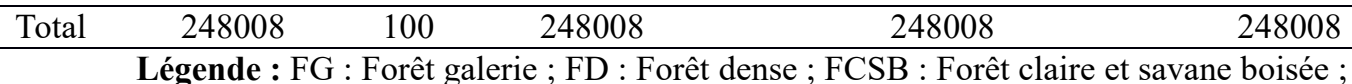

SAA : Savanes arborée et arbustive ; SS : Savane saxicole ; PT : Plantation ; MCJ :

Mosaïque de Champs et jachères ; AGG : Agglomération ; Tx : Taux d'expansion entre 2016 et 2031 en $\%$

A la lecture de la figure 3 et du tableau III, il ressort que dans le scénario « Business-As-Usual (BAU) 》 (scénario de référence), les forêts denses, les forêts claires et savanes boisées et les savanes arborées et arbustives vont diminuer respectivement de $67,7 \% ; 51,6 \%$; et $45,9 \%$ d'ici à 2031 par rapport à l'année de référence 2016. La superficie des plantations, des mosaïques de cultures et jachères et des agglomérations vont augmenter respectivement de $60,4 \% ; 37,6 \%$ et de $21,5 \%$ dans la période simulée. Les superficies des forêts denses, des forêts claires et savanes boisées et des savanes arborées et arbustives vont considérablement transformées en mosaïques de cultures et jachères. Ainsi, la conversion de ces terres en terres cultivées devrait se produire principalement dans les parties moins accidentées au nord-ouest et sud-est du secteur d'étude.

Dans le scénario de durabilité environnementale coordonnée (DEC) ou «Alafia », nous avons supposé que l'utilisation du sol est réglementée par une politique de protection de l'environnement au cours de la période 2016-2031. Les forêts denses et les savanes arborées et arbustives ont régressé respectivement de seulement de 33,2\% et de 44,2 \%. Quant aux forêts claires et savanes boisées, elles ont connu une expansion de $02,6 \%$. Les plantations, les mosaïques de cultures et jachères et les agglomérations augmenteront respectivement de $145 \%, 34,3 \%$ et $04,9 \%$. L'expansion de la superficie de forêts claires et savanes boisées proviendrait principalement des savanes arborées et arbustives.

Dans ce scénario durabilité environnementale coordonnée (DEC), la zone de forêts claires et savanes boisées a augmenté de manière significative $(02,6 \%)$ tandis que le taux de déclin des forêts denses et des savanes arborées et arbustives est légèrement inférieur à celui des deux autres scénarios (BAU et CER). Ceci témoigne que la politique de protection de l'environnement jouerait un rôle positif dans la conservation des ressources végétales naturelles. De plus, cette politique a encouragé à la fois l'agriculture et une augmentation de la zone sylvicole. Dans le scénario de croissance économique rapide (CER), les plantations, les mosaïques de cultures et jachères et les agglomérations vont évoluer de façon considérable (exagérée) respectivement de $60 \% ; 45,7 \%$ et $40 \%$. La conséquence est une régression plus accentuée des formations végétales naturelles que celle observée au niveau des scénario BAU et DEC.

Dans ces trois scénarii, la superficie augmentée au niveau des plantations proviendrait majoritairement des savanes arborée et arbustives et 
des mosaïques de cultures et jachères. L'expansion de la zone bâtie (agglomération) proviendrait principalement de la conversion des mosaïques de cultures et jachères. Ces dernières doivent leur expansion aux zones de forêts denses, de forêts claires et savanes boisées, de savanes arborées et arbustives.

\section{Calcul du LUCC budget pour la transition 2016-2031}

Les matrices de transition extraites entre la carte d'occupation réelle de 2016 et celles simulées à l'horizon 2031 pour les trois (03) différents scenarii ont permis de faire le LUCC budget entre 2016 et 2031 (tableaux IV, $\mathrm{V}$ et VI). Le LUCC budget a permis de mieux apprécier les flux d'échange de catégories d'occupation des terres qui s'opéraient entre 2016 et 2031.

Tableau IV : LUCC Budget (2016-2031) par rapport au scénario BAU

\begin{tabular}{cccccc}
\hline & Gain & Perte & $\begin{array}{c}\text { Changement } \\
\text { total }\end{array}$ & Balance & $\begin{array}{c}\text { valeur absolue } \\
\text { changement net }\end{array}$ \\
\hline FG & 0 & 0 & 0 & 0 & 0 \\
\hline FD & 0 & 392,3 & 392,3 & 0 & 392,3 \\
\hline FCSB & 0 & 2124,6 & 2124,6 & 0 & 2124,6 \\
\hline SAA & 0 & 46126,3 & 46126,3 & 0 & 46126,3 \\
\hline SS & 0 & 366,1 & 366,1 & 0 & 366,1 \\
\hline PT & 644,5 & 0 & 644,5 & 0 & 644,5 \\
\hline MCJ & 48873,6 & 1676 & 50549,6 & 3352 & 47197,6 \\
\hline AGG & 1167,2 & 0 & 1167,2 & 0 & 1167,2 \\
\hline & 50685,3 & 50685,3 & 101370,6 & 3352 & 98018,6 \\
\hline
\end{tabular}

Légende : FG : Forêt galerie ; FD : Forêt dense ; FCSB : Forêt claire et savane boisée ;

SAA : Savanes arborée et arbustive ; SS : Savane saxicole ; PT : Plantation ; MCJ :

Mosaïque de Champs et jachères ; AGG : Agglomération

Tableau V : LUCC budget (2016-2031) par rapport au scénario DEC

\begin{tabular}{cccccc}
\hline & Gain & Perte & $\begin{array}{c}\text { Changement } \\
\text { total }\end{array}$ & Balance & $\begin{array}{c}\text { valeur absolue } \\
\text { changement net }\end{array}$ \\
\hline FG & 0 & 0 & 0 & 0 & 0 \\
\hline FD & 188 & 380,4 & 568,6 & 375,8 & 192,5 \\
\hline FCSB & 2631,2 & 2521,5 & 5152,7 & 5043,1 & 109,6 \\
\hline SAA & 1744,4 & 46197,5 & 47941,8 & 3488,8 & 44453,1 \\
\hline SS & 30,3 & 359,5 & 389,8 & 60,7 & 329,1 \\
\hline PT & 1548,2 & 0 & 1548,2 & 0 & 1548,2 \\
\hline MCJ & 45256,8 & 2203,4 & 47460,2 & 4406,8 & 43053,4 \\
\hline AGG & 263,5 & 0 & 263,5 & 0 & 263,5 \\
\hline & 51662,3 & 51662,3 & 103324,5 & 13375,1 & 89949,4 \\
\hline
\end{tabular}

Légende : FG : Forêt galerie ; FD : Forêt dense ; FCSB : Forêt claire et savane boisée ;

SAA : Savanes arborée et arbustive ; SS : Savane saxicole ; PT : Plantation ; MCJ :

Mosaïque de Champs et jachères ; AGG : Agglomération 
Tableau VI : LUCC budget (2016-2031) par rapport au scénario CER

\begin{tabular}{cccccc}
\hline & Gain & Perte & $\begin{array}{c}\text { Changement } \\
\text { total }\end{array}$ & Balance & $\begin{array}{c}\text { valeur absolue } \\
\text { changement net }\end{array}$ \\
\hline FG & 0 & 0 & 0 & 0 & 0 \\
\hline FD & 0 & 392,3 & 392,3 & 0 & 392,3 \\
\hline FCSB & 0 & 1894,3 & 1894,3 & 0 & 1894,3 \\
\hline SAA & 0 & 57494,3 & 57494,3 & 0 & 57494,3 \\
\hline SS & 0 & 348,3 & 348,3 & 0 & 348,3 \\
\hline PT & 641,3 & 0 & 641,3 & 0 & 641,3 \\
\hline MCJ & 59904,6 & 2588 & 62492,6 & 5176 & 57316,7 \\
\hline AGG & 2171,3 & 0 & 2171,3 & 0 & 2171,3 \\
\hline & 62717,2 & 62717,2 & 125434,4 & 5176 & 120258,5 \\
\hline
\end{tabular}

Légende : FG : Forêt galerie ; FD : Forêt dense ; FCSB : Forêt claire et savane boisée ;

SAA : Savanes arborée et arbustive ; SS : Savane saxicole ; PT : Plantation ; MCJ :

Mosaïque de Champs et jachères ; AGG : Agglomération

De l'examen des tableaux IV et VI aucune formation végétale naturelle n'a gagné de superficie en 2031. En effet, au niveau de ces deux tableaux qui présentent respectivement le LUCC budget (2016-2031) par rapport au scénario BAU et au scénario CER, les forêts denses, les forêts claires et savanes boisées, les savanes arborées et arbustives et les savanes saxicoles perdront d'importantes superficies contre zéro gagnée. Seules les plantations, les mosaïques de cultures et jachères et les agglomérations gagneront des superficies à l'horizon 2031. Par contre les mosaïques de cultures et jachères, ont aussi bien perdues que gagnées de superficie. Soit un changement net de 37,6 \% (47197,6 ha) par rapport au scénario BAU et 45,7 \% (5 7316,7 ha) par rapport au scénario CER. Les galeries forestières sont restées presque stable. On note clairement dans ce scénario une dégradation continue des formations végétales naturelles au profit des formations anthropiques.

De l'analyse du tableau V, il se dégage qu'en 2031 contrairement au deux autres scénarii, le LUCC budget par rapport au scénario DEC montre un gain de superficies des forêts denses, des forêts claires, des savanes boisées et des savanes arborées et arbustives.

Du coup, globalement les forêts denses, les forêts claires et savanes boisées et les savanes arborées et arbustives ont perdu respectivement 33,2\% (192,5 ha), 2,7\% (109,2 ha) et 44,2\% (44 453,1 ha) de leur superficie en 2031. Les plantations ont gagné $01,4 \%$ (1548,2 ha) et les agglomérations ont gagné $04,9 \%$ (263,5 ha). Les superficies gagnées par les forêts claires et savanes boisées proviennent de la dégradation des forêts denses et de l'évolution d'une partie des savanes boisées et arbustives. Quant aux superficies gagnées par les savanes arborées et arbustives, elles proviendraient de la dégradation des forets claires et savanes boisées, et beaucoup plus des mosaïques de cultures et jachères. Les superficies gagnées par les plantations et les agglomérations proviendraient des savanes arborées et arbustives des mosaïques de cultures et 
jachères.

\section{Comparaison des cartes simulées des trois scénarios BAU, DEC et CER}

L'étude comparée des scénarios Business-As-Usual (BUA), durabilité environnementale coordonnée (DEC) et croissance économique rapide (CER) permet de constater que dans l'ensemble des trois scénarios, le scénario CER est le plus sévère avec une occupation de plus de $70 \%$ de la zone d'étude par les mosaïques de cultures et jachères. Cette catégorie d'occupation des terres est concentrée beaucoup plus dans le nord-ouest et le sud-est. Ce sont des zones moins accidentées et relativement plates, présentant des aptitudes aux cultures vivrières. La figure 4 présente la carte de l'étude croisée des trois différents scénarios BAU, DEC et CER.

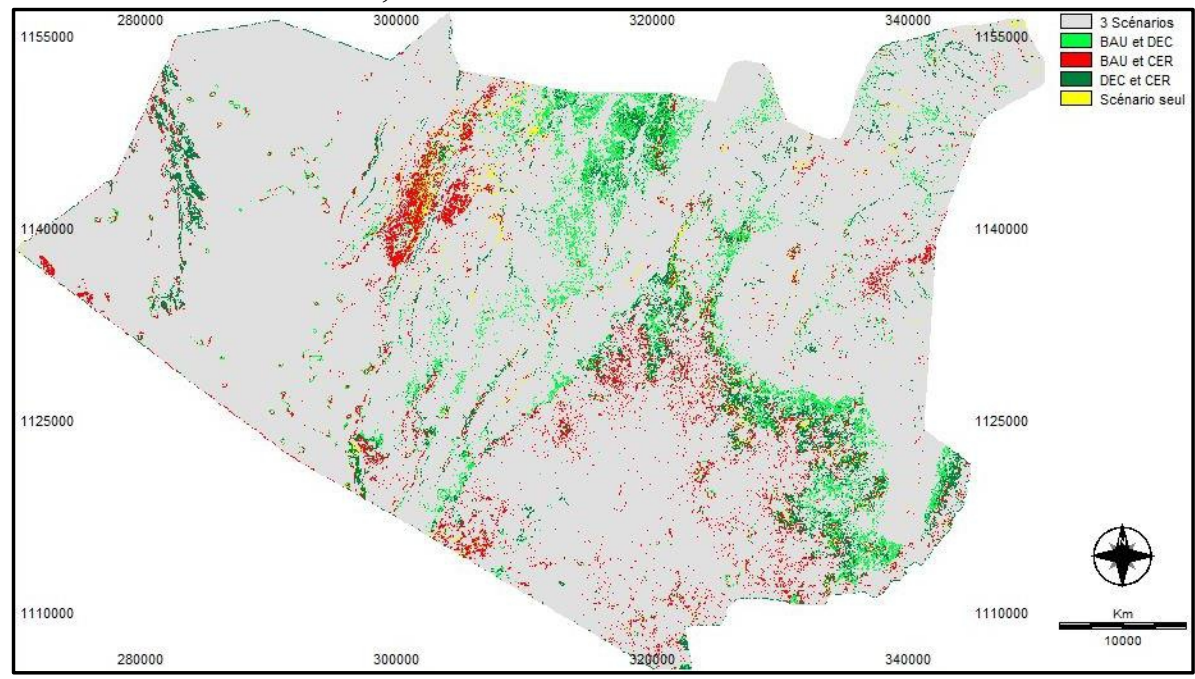

Figure 4 : Comparaison des trois scénarios BAU, DEC et CER

Légende : 3 Scénarios : Superficie commune aux scénarios BAU, DEC et CER ;

BAU et DEC : Superficie commune aux scénarios DEC et CER ;

BAU et CER : Superficie commune aux scénarios BAU et CER;

DEC et CER : Superficie commune aux scénarios DEC et CER;

Scénario seul : Superficie non commune aux différents scénarios.

Pour mieux analyser cette figure, les statistiques des différentes intersections identifiées entre les trois scénarios BAU, DEC et CER ont été extraites (tableau VII).

Tableau VII : Proportion des superficies occupées par chaque catégorie d'occupation des terres dans les trois scénarios

\begin{tabular}{|c|c|c|c|c|c|c|c|c|}
\hline & \multicolumn{7}{|c|}{ Proportion en \% de la superficie totale des 3 scénarii } & \\
\cline { 2 - 9 } & $\begin{array}{c}\text { Intersection } \\
\text { des 3 } \\
\text { scénarios }\end{array}$ & $\begin{array}{c}\text { Intersection } \\
\text { BAU et DEC }\end{array}$ & $\begin{array}{c}\text { Intersection } \\
\text { BAU et CER }\end{array}$ & $\begin{array}{c}\text { Intersection } \\
\text { DEC et CER }\end{array}$ & $\begin{array}{c}\text { BAU } \\
\text { seul }\end{array}$ & $\begin{array}{c}\text { DEC } \\
\text { seul }\end{array}$ & $\begin{array}{c}\text { CER } \\
\text { seul }\end{array}$ & $\begin{array}{c}\text { Total } \\
\text { en \% }\end{array}$ \\
\hline FG & 100 & 0 & 0 & 0 & 0 & 0 & 0 & $\mathbf{1 0 0}$ \\
\hline FD & 14,1 & 16,2 & & 39,8 & 0 & 15,8 & 14,1 & $\mathbf{1 0 0}$ \\
\hline
\end{tabular}




\begin{tabular}{|c|c|c|c|c|c|c|c|c|}
\hline FCSB & 35,2 & 3,5 & 2,3 & 22,1 & 0,2 & 11,2 & 25,6 & $\mathbf{1 0 0}$ \\
\hline SAA & 76,9 & 15,9 & 1,7 & 3,1 & 0,9 & 1,4 & 0,1 & $\mathbf{1 0 0}$ \\
\hline SS & 97,5 & 0 & 0,1 & 2,3 & 0 & 0,1 & 0 & $\mathbf{1 0 0}$ \\
\hline PT & 53,5 & 0,8 & 0,1 & 25 & 6 & 8,1 & 6,6 & $\mathbf{1 0 0}$ \\
\hline MCJ & 91,3 & 0,5 & 4,1 & 3,3 & 0,5 & 0,1 & 0,2 & $\mathbf{1 0 0}$ \\
\hline AGG & 84,7 & 0 & 12,7 & 0,0 & 0,0 & 0 & 2,5 & $\mathbf{1 0 0}$ \\
\hline $\begin{array}{l}\text { Total } \\
\text { en \% }\end{array}$ & $\mathbf{8 8 , 4}$ & $\mathbf{3 , 8}$ & $\mathbf{3 , 6}$ & $\mathbf{3 , 6}$ & \multicolumn{3}{|c|}{$\mathbf{0 , 6}$} & \\
\hline
\end{tabular}

Légende : FG : Forêt galerie ; FD : Forêt dense ; FCSB : Forêt claire et savane boisée ;

SAA : Savanes arborée et arbustive ; SS : Savane saxicole ; PT : Plantation ; MCJ :

Mosaïque de Champs et jachères ; AGG : Agglomération ;

BAU : Business As Usual ; DEC : Durabilité Environnementale Coordonnée ; CER :

Croissance Economique Rapide

De l'examen de la figure 4 et du tableau VII, il ressort que $88,4 \%$ de la superficie de la zone d'étude (219319,3 ha) est commun aux trois scenarii de simulation. L'intersection entre BAU et DEC, entre BAU et CER et entre DEC et CER est estimée respectivement à 3,8 \% (9425,7 ha) ; 3,01\% (8987,7 ha) et 3,6\% (8840,6 ha). Les superficies qui diffèrent individuellement selon le scénario considéré ne représentent que $0,6 \%$ (1457,6 ha). Les superficies qui ont le plus variées en fonction du scénario choisi sont les forêts denses et les plantations. Les proportions de ces catégories d'occupation des terres sont plus importantes dans le scénario DEC (entre 8,1 et 15,8\%) que dans les autres scénarios BAU et CER (entre 0 et 14,1\%). Quant aux forêts claires et savanes boisées, $25,6 \%$ de leurs superficies sont retrouvées uniquement dans le scénario CER, alors qu'elles n'occupent que $0,9 \%$ de la superficie du milieu d'étude à l'horizon 2031 dans ce scénario.

Globalement pour les trois scénarios, le scénario CER est plus sévère avec une occupation de plus de $70 \%$ de la zone d'étude par les mosaïques de cultures et jachères. Cette catégorie d'occupation des terres est concentrée beaucoup plus dans le nord-ouest et le sud-est. Ce sont des zones les moins accidentées et relativement plates, présentant des aptitudes aux cultures vivrières.

\section{Discussion}

Pendant de longue durée, les forêts denses, forêts claires et savanes boisées de la région sont détruites et les terres mises en culture. Dès que de faibles rendements agricoles commencent par être enregistrés, ces terres sont mises en jachères. Souvent ces jachères ne reviennent plus à la forêt mais sont réinsérés dans une rotation de champs cultivés ou sont transformées en savanes arborées ou arbustives simplement. Ceci corrobore les résultats obtenus par Bidou et al. (2013). Ces mêmes auteurs avaient démontré dans leur étude qu'au nord-ouest du Bénin, les gros hameaux qui se trouvent sur les routes, et leurs marchés jouent un rôle aussi important dans le développement 
de l'agriculture que la seule croissance démographique.

En résumé, la tendance de régression du paysage ligneux au profit de la culture et des espaces urbains observée depuis 1987 a été aussi observée par plusieurs auteurs dont, Leroux (2012) et Bidou et al. (2013) qui ont réalisé des études sur le Bénin et dans la région soudanienne. Celle-ci se fait alors aux dépens des centres urbains et villageois mais également le long des axes de communication principaux (réseau de routes, réseau de pistes). Par ailleurs, cette dégradation impact surtout les espaces de végétations denses. L'utilisation de ces espaces déboisés permet de bénéficier de nouvelles terres fertiles et donc d'augmenter les productions agricoles. Du coup, la déforestation entraîne une perte de la biodiversité en raison de la destruction de nombreux habitats naturels (Toko, 2008 ; Arouna, 2012).

Les différents scénarios prospectifs conçus ici tiennent compte des différentes activités socio-économiques développées dans le milieu d'étude. De façon globale, la tendance évolutive des formations végétales dans cette étude est régressive et celle des formations anthropiques évoluent de façon progressive. Dans ce scénario BAU, les superficies des mosaïques de cultures et jachères, des agglomérations et des plantations ont augmenté. L'augmentation de ces catégories d'occupations des terres pourrait s'expliquer par l'augmentation de la population et donc des besoins de nourriture et de logement. En compensation à cette forte demande de terres, il y a une réduction des superficies de savanes. Ceci concorde bien avec les travaux de Abdus et al. (2017) mené sur Punjab une province du Pakistan et qui explique la forte demande des terres cultivées et du bâti par l'augmentation de la population. Le scénario catastrophique (CER) prévoit que les formations végétales naturelles vont régressées aux profit de celles anthropiques. Ces prévisions concordent avec celles de Mama (2002) à l'horizon 2025 pour les Communes de Savè, Ouèssè et Tchaourou et celle de Oloukoï (2013) pour la même zone mais à l'horizon 2034. Ces résultats concordent aussi avec ceux de Orekan (2007) qui a montré avec le modèle Clue's que dans la région centrale du Bénin d'ici 2025 d'importance superficie de forêts vont disparaitre au profit des mosaïques de cultures et jachères. Le scénario durabilité environnementale coordonnée (DEC) qui combine à la fois la préservation du couvert végétal avec les activités agricoles responsabilise l'Etat dans son rôle de contrôle de la déforestation et de subvention du gaz domestique pour remplacer le bois de feu. Ceci est similaire au scénario de densification développé par Lajoie et Hagen-Zanker (2007) qui encourage la préservation des forêts et limite l'étalement urbain dans l'Ile de la Réunion à l'horizon 2031.

\section{Conclusion}

Ce milieu a connu une dynamique spatiale importante entre 1987 et 
2016. Les changements observés s'opèrent de façon différente au cours des périodes 1987-2000 et 2000-2016. De façon globale, ce milieu a vu ces formations végétales naturelles notamment les forêts denses, forêts claires et savanes boisées se réduire au profit des formations anthropiques comme les mosaïques de cultures et jachères, les plantations et les agglomérations. Cette dynamique spatiale du paysage s'explique surtout par les pressions anthropiques.

L'approche prospective utilisée dans cette étude a permis de comprendre et de montrer le devenir des territoires à l'horizon 2031. Le modèle dynamique LCM utilisé pour la simulation permet d'observer des changements dans l'occupation du sol en fonction du scénario utilisé. Il est à retenir que, quel que soit le scénario, la superficie des mosaïques de cultures et jachères, des zones bâties et des plantations sont en augmentation. Ce paysage dont dépend la survie des populations évolue dans le sens de sa dégradation. Et sans inversion de la tendance actuelle, la situation s'aggravera d'ici 2031 et pourrait devenir irréversible. Cependant les résultats obtenus avec le scénario Durabilité Environnementale Coordonnée (DEC) montrent que l'espoir est permis. Au-delà de l'exploitation des ressources ligneuses et de l'expansion des terres agricoles, pour améliorer la sécurité alimentaire, il est indispensable de mettre en place des stratégies de gestion durable des ressources naturelles de ce milieu. Les décideurs et acteurs en charge de la gestion territoriale doivent donc prendre en compte ces résultats dans leurs prises de décisions.

\section{References:}

1. Abdus S., Xiangzheng D., Siqi J. and Dongdong C. (2017). ScenarioBased Simulation on Dynamics of Land-Use-Land-Cover Change in Punjab Province, Pakistan. Sustainability 2017, 9, 1285. 17 p.

2. Akognongbé A., Abdoulaye D., Vissin E.W. et Boko M. (2014). Dynamique de l'occupation du sol dans le bassin versant de l'Oueme à l'exutoire de Bétérou (Bénin). Afrique Science 10(2) (2014) 228 242

3. Arouna O. (2012). Cartographie et modélisation prédictive des changements spatio-temporels de la végétation dans la Commune de Djidja au Bénin : implications pour l'aménagement du territoire. Thèse de Doctorat Unique Université d'Abomey-Calavi, Bénin, 246 p.

4. Bamba I., Mama A., Neuba D. F. R., Koffi K. J., Traoré D., Visser M., Sinsin B., Lejoly J. et Bogaert J. (2008). Influence des actions anthropiques sur la dynamique spatio-temporelle de l'occupation du sol dans la province du Bas-Congo (R.D. Congo). Sciences \& Nature Vol. $5 \mathrm{~N}^{\circ} 1: 49-60$. 
5. Bidou E. J., Palibrk M., Andrieu J., Mering C. (2013). Vulnérabilité, dynamique de population et occupation des sols : Réflexion sur les indicateurs de vulnérabilité au Bénin et au Niger. Livrable nº.4 Février, 28 pages.

6. Bigot S., Zin I., Diedhiou A. (2005). Apports de données de HRV de SPOT pour l'étude des variations phénologiques dans le bassin de l'Ouémé (Bénin). Télédétection. Vol 4(4), pp 339-353.

7. FAO (2016). Situation des forêts du monde. Forêts et agriculture : défis et possibilité concernant l'utilisation des terres. Rome (Italie), $137 \mathrm{p}$. ISBN 978-92-5-209208-7

8. Faye E., Masse D. et Diatta M. (2002). Dynamique de la régénération ligneuse durant la phase de culture dans un système de culture semipermanente du sud du Sénégal. Actes du colloque, mai 2002, Garoua, Cameroun. Prasac, N'Djamena, Tchad-Cirad, Montpellier, France.

9. Houssou C. (1998). Les bioclimats humains de l'Atacora (Nord-ouest du Bénin) et leurs implications socio-économiques. Thèse de Doctorat (Thèse unique). Dijon, France, $332 \mathrm{p}$.

10. Jacquin A. (2010). Dynamique de la végétation des savanes en lien avec l'usage des feux à Madagascar. Analyse par séries temporelle d'images de télédétection. Thèse de Doctorat de l'université de Toulouse. France, 144 p.

11. Lajoie, G. et Hagen-Zanker, A. (2007). La simulation de l'étalement urbain à la Réunion. Apport de l'automate cellulaire pour la prospective territoriale. Cybergeo : European Journal of Geography [En ligne], Systèmes, Modélisation, Géostatistiques.

12. Leroux L. (2012). Analyse diachronique de la dynamique paysagère sur le bassin supérieur de l'ouémé (Bénin) à partir l'imagerie LandsatMODIS- cas d'étude du communal de Djougou. Hydrosciences Montpellier (France), ANR ESCAPE, 62.

13. Mama V.J. (2002). An integrated approach for land-use/cover change analysis in a central region of Benin Republic. PhD Thesis, Geography, University of Lagos, Lagos, $221 \mathrm{p}$.

14. Oloukoi J. (2013). Scénario socio-économique et écologique des changements de l'occupation des terres au Bénin », Vertigo - la revue électronique en sciences de l'environnement [En ligne], Volume 13 Numéro 1/avril 2013, mis en ligne le 16 avril 2013. URL : http://vertigo.revues.org/13267 ; DOI : 10.4000/vertigo.13267, consulté le 02 février 2014.

15. Orékan V. O. A. (2007). Implémentation du modèle CLUE-s aux transformations spatiales dans le centre Bénin aux moyens de données socio-économiques et de télédétection. Thèse de Doctorat de l'université de Bonn. Allemagne, $230 \mathrm{p}$. 